S0009-2509(96)00121-2

\title{
MICROMIXING EFFECTS IN A STIRRED TANK: THE RANDOM REPLACEMENT IEM MODEL
}

\author{
P. C. Chang", C. Y. Mou' and D. J. Lee ${ }^{b^{*}}$ \\ 'Department of Chemistry \\ bDepartment of Chemical Engineering, National Taiwan University, Taipei, Taiwan, 106, ROC
}

\begin{abstract}
A random replacement IEM model, in which the mean field concentration is considered as a direct averaging over all the existing fluid particles in the stirred tank, is proposed as a numerically more favourable IEM scheme. For steady-state simulations, the present random replacement IEM model is equivalent to the steady-state IEM model. For chemical systems with a stiff kinetics, the random replacement IEM model is found superior to the unsteady-state IEM model. Two numerical examples are given.
\end{abstract}

\section{INTRODUCTION}

Stirring may alter the complex system dynamics both quantitatively and qualitatively (Villermaux, 1991, Dutt and Menzinger, 1992). The importance of mixing is usually discussed in terms of macromixing and micromixing. The effects of incomplete macromixing on complex chemical dynamic systems have been investigated by several investigators (for a brief review, see Hsu et al., 1994, 1995). Zonal models are widely employed. On the other hand, the role of micromixing is generally studied based on the micromixing models (Horsthem and Hannon, 1984, Puhl and Nicolis, 1986, 1987, Boissonade and DeKepper, 1987, Fox and Villermaux, 1990a, 1990b, Villermaux, 1991, Gyorgyi and Field, 1992, Chang, 1992, Rouff, 1993, Fox et al., 1994). A brief but not complete summary is given in Table 1. Clearly, the most widely employed micromixing models are the coalescence-dispersion (CD) model and the interaction-by-exchange-with-the-mean (IEM) model. The latter had recently received great research interest (Fox et al., 1994).

In the IEM model, each fluid particle has entered the reactor is assumed to exchange manner with the mean fluid field with a characteristic time of $t_{m}$. The completeness of micromixing can thereby be discussed by varying $t_{m}$. The IEM model has been successfully employed in various chemical systems, including the classical chlorite-iodide bistable/oscillatory systems (Fox and Villermaux, 1990b, Fox et al., 1994). However, as demonstrated latter, the existing numerical scheme is not suitable for the systems with a stiff time evolution, such as the Oregonator. In these cases, a very large number of fluid particles may be required in simulations to make the fluid particle inflow/outflow exchange time comparable to the characteristic time of the reaction kinetics.

The focus of this report is to propose a numerically more favourable IEM scheme. Two examples are given for demonstrating the applicability of the proposed model.

\section{THE IEM MODEL}

The mass balance for the fluid particle of age $\alpha$ entering at inlet port $\mathrm{j}$ is stated as:

$$
\frac{d \mathbf{C}_{i}^{(j)}(\alpha)}{d t}=\frac{1}{t_{m}}\left(<\mathbf{C}>-\mathbf{C}_{i}^{(j)}(\alpha)\right)+\mathbf{R}(\mathbf{C}(\alpha))
$$

where $C_{i}^{\omega}$ denotes the concentration vector in the $\mathrm{i}$-th fluid particle entering at port $\mathrm{j}, t_{m}$ the mixing time, and $\mathbf{R}$ the reaction term. In a perfectly macro-mixed CSTR, the residence time of the fluid particles follows an exponential distribution. The mean field concentration $\langle\mathrm{C}\rangle$ is thereby defined as follows:

$$
<\mathrm{C}>=\Sigma_{j} \frac{\beta_{j}}{\tau} \int_{0}^{\infty} C_{i}^{(j)}(\alpha) e^{-\alpha \tau} d \alpha,
$$


Table 1. Some micromixing studies in the literature.

\begin{tabular}{|c|c|c|}
\hline Ref & chemical system & model \\
\hline Horsthemke and Hannon (1984) & Schogl model & $\mathrm{CD}$ \\
\hline Puhl and Nicolis (1986) & Gray-Scott & Zwietering micromixing \\
\hline Puhl and Nicolis (1987) & Gray-Scott & Zwietering micromixing \\
\hline Boissonade amd DeKepper (1987) & Kumpinsky-Epstein & CD/IEM \\
\hline Fox and Villermaux (1990a) & Citri-Epstein & IEM \\
\hline Fox and Villermaux (1990b) & Citri-Epstein & IEM \\
\hline Villermaux (1991) & Brusselator & IEM \\
\hline Gyorgyi and Field (1992) & minimal $\mathrm{BrO}_{3}^{-}$oscillator & segregate-feed \\
\hline Chang (1992) & Oregonator & IEM \\
\hline Ruoff (1993) & Oregonator & modified IEM \\
\hline Fox et al. (1994) & Citri-Epstein & IEM \\
\hline
\end{tabular}

IEM: interaction-by-exchange-with-the-mean model.

CD: coalesence-dispersion model.

Zwietering: Zwietering model

where $\beta_{j}$ denotes the portion of fluid flowing from port $\mathrm{j}, \tau$ the residence time of the reactor, and $\alpha$ the time the fluid particle had experienced after entering the reactor. Clearly eq. (2) is a weighted average of fluid particle concentrations based on the probability of the presence of the fluid particle in the stirred tank.

Eqs. (1) and (2) are self-consistent and form the basis of the steady-state IEM (SSIEM) model. The iterations between eqs. (1) and (2) can be employed for finding the steady states for the chemical systems under incomplete micromixing condition. A small $t_{m}$ indicates a well micro-mixed medium.

Assume that the fluid particle had entered at time $t-\alpha$ and had spent an time interval of $\alpha^{\prime}$ in the reactor. To extend to the unsteady state applications, the unsteady state IEM model has been formulated based on the following transient mass balance equation of the mean field concentration $\langle\mathrm{C}\rangle$ (Fox and Villermaux, 1990a):

$$
\left[\frac{\partial \mathbf{C}_{i}^{(j)}\left(\alpha^{\prime}, t-\alpha\right)}{\partial \alpha^{\prime}}\right]_{\alpha^{\prime}=\alpha}=R\left(\mathbf{C}_{i}^{(j)}(\alpha, t-\alpha)\right)+\frac{1}{t_{m}}\left(\langle\mathbf{C}(t)\rangle-\mathbf{C}_{i}^{(j)}(\alpha, t-\alpha)\right) .
$$

Clearly, we have the following equality:

$$
\frac{d \mathbf{C}_{i}^{(j)}(\alpha, t-\alpha)}{d \alpha}=\left[\frac{\partial \mathbf{C}_{i}^{(j)}\left(\alpha^{\prime}, t-\alpha\right)}{\partial \alpha^{\prime}}\right]_{\alpha^{\prime}=\alpha}-\frac{d \mathbf{C}_{i}^{(j)}(\alpha, t-\alpha)}{d t} .
$$

Take a weighted average for both sides of eq. (4) with the operator $\sum_{j} \frac{1}{\tau} \int_{0}^{\infty}(\bullet) \exp (-\alpha / \tau) d \alpha$, the following equation can be obtained by integration by parts and with the basic definition of mean field concentration (eq. (2)) and eq. (3):

$$
\frac{d<\mathbf{C}>}{d t}=\Sigma_{j} \beta_{j} \int_{0}^{\infty} \frac{1}{\tau} \mathbf{R}\left[\mathbf{C}_{i}^{(j)}(\alpha, t-\alpha)\right] e^{-\alpha / \tau} d \alpha+\frac{1}{\tau}\left(\mathbf{C}_{0}-<\mathbf{C}>\right)
$$

The coupling between eqs. (1) and (5) forms the so-called unsteady-state IEM (USIEM) model. (Fox and Villermaux, 1990a, 1990b, Fox et al., 1994). Notably eq. (5) contains no parameter $t_{m}$. Equations. (1) and (5) are also self-consistent from which the time evolution path of $\langle\mathrm{C}>$ could be subsequently obtained.

In numerical practice, nevertheless, the accurate evaluation of integrand in eq. (5) for USIEM model is essential for the evaluation of the evolution path of the $\langle\mathrm{C}\rangle$. Simple algorithms such like the Simpson's rule can be easily employed for non-stiff systems. However, the accuracy of numerical evaluations of these integrands would be a consequential problem when the reaction term becomes stiff. A very large number of fluid particles may require in simulation for giving a fluid particle inflow/outflow exchange time comparable to the reaction characteristic time. This restriction has put an obstacle to the applicability of the IEM model in investigation of micromixing effects.

\section{THE RANDOM REPLACEMENT IEM MODEL}

The newly proposed model is called random replacement IEM model, which adopts the scheme similar to the molecular dynamics (MD) simulation. In a perfectly macro-mixed CSTR, the probability for all the particles existing in the tank at time $t$ to leave the tank should be equal. Therefore, if repeatedly, randomly replacing a particle in the tank regardless of its age with a new particle of an age 0 at a time interval of $\Delta \alpha(=\tau / \mathrm{N})$, a stationary residence time distribution of exponential-type $(\exp (-\alpha / \tau) / \tau)$ will be asymptotically approached no matter what is 
the initial life time distribution of particles. With such a stationary distribution, we can thereby work on the absolute time scale, while the average concentration over the whole tank can be taken as:

$$
<\mathbf{C}>=\frac{1}{N} \sum_{j} \Sigma_{i} \beta_{j} \mathbf{C}_{i}^{(j)}(t)
$$

Note that in eq. (6) the mean field concentration $<\mathbf{C}>$ is evaluated based on all fluid particles present in the stirred tank at time $t$ regardless of their age $\alpha$. Equations (1) and (6) are self-consistent and are referred to as the "random replacement IEM model" in this paper.

Notably the integration term of eq. (2) can be approximated by the following finite summation if there exists a finite number of fluid particles whose age is between $(\alpha, \alpha+\Delta \alpha)$ :

$$
<\mathbf{C}>\cong \Sigma_{j} \beta_{j} \sum_{i} \mathbf{C}_{i}^{(j)}(\alpha) \exp (-\alpha / \tau) \Delta \alpha / \tau
$$

It is therefore clear that since $\Delta \alpha=\tau / N$ and since the number of the existing particles in the tank of age between $(\alpha, \alpha+\Delta \alpha)$ is $N \exp (-\alpha / \tau) \Delta \alpha / \tau$, the average in eq. (6) is essentially equivalent to eq. (7), or eq. (2) for SSIEM, if $N$ is large enough.

For the unsteady- state applications, it is interesting to have a closer look at eqs. (3)-(5) for the derivation of the USIEM model. Equation (3) is the transient mass balance of a fluid particle, which is a material description of the reactant concentrations. Equation (4) has transformed the description based on a fixed coordinate. By taking the weighted average based on the residence time distribution, eq. (5) results. It is noted that the derivation of eq. (5) is still based on the basic definition of mean field concentration, eq. (2). We can therefore still use eqs. (1) and (2), or equivalently, eqs. (1) and (6) in the unsteady-state cases. There is therefore no difference between steady-state and unsteady-state applications from the view point of the random replacement IEM model.

Although the proposed random replacement IEM model is equivalent to conventional IEM model when fluid particle number is large, the former model has avoided the necessity for numerical evaluation of the integrand in eqs. (2) or (5) for SSIEM or USIEM models. Reaction term appears only in eq. (1), which can be easily handled by an implicit ODE solver. The modelling capability on systems with a stiff time revolution should therefore be largely enhanced for the random replacement IEM model

\section{RESULTS AND DISCUSSION}

For demonstration purpose, two examples will be given in the following sections: (a) the Kumpinsky-Epstein (K-E) model, which exhibits bistable characteristics under certain parameter range (Kumpinsky and Epstein, 1985, Hsu and Lee, 1995), and (b) the three-variable, irreversible Oregonator model (abbr. as Oregonator hereafter), which exhibits simple oscillatory characteristics (Field and Noyes, 1974, Hsu et al., 1994). The former would be employed for discussion on the differences between the SSIEM and the random replacement IEM model. While for the latter, the results from USIEM model and the random replacement IEM model are compared. For simplicity, only the cases with one input port are considered.

\section{Kumpinsky-Epstein Model}

The K-E model and the corresponding parameters can be found elsewhere (Hsu and Lee, 1995). The simulation results by the random replacement IEM model are demonstrated in Fig. 1 . An increase in mixing time, $t_{m}$, would shift the location and the transition point for the thermodynamic branch towards the region of higher $Y$ and larger $\tau$, but has almost no effect on the flow branch. This is consistent with the experiments (Menzinger et al., 1986).

The present simulation results are found to agree well with those obtained from the original SSIEM model. This supports the arguments that the random replacement IEM model is equivalent to the SSIEM model when the particle number is large enough. However, the computer execution time is still somewhat less for the former model.

\section{The Oregonator Model}

The Oregonator model and the corresponding parameters can be found elsewhere (Hsu et al., 1994). The parameters employed would give a relaxation oscillation behaviour with four essential stages within a cycle. The stiffest part of oscillation is the stage where the concentration of reactant $Y$ suddenly drops.

Figure 2 shows the time evolution of the mean field concentration of $Y$ with 1000 fluid particles $(N=1000)$. Simulation results clearly show the four essential stages in an oscillation cycle for various $t_{m}$ values. The very stiff, sudden drop in concentration of $\mathrm{Y}$ are easily generated (denoted by symbol $\mathrm{X}$ in the figure). Notably, the evolution paths for $t_{m}=0.2$ and 1.0 are close. That is, the tank can be taken as well micro-mixed when mixing time is less than approximately 1.0. The oscillation period is increased as $t_{m}$ decreases (better mixing). This is qualitatively consistent with the experiments (Menzinger et al., 1986) and is parallel to that found for the incomplete macromixing (Hsu et al., 1994). The calculated oscillation periods with two fluid particle numbers are 


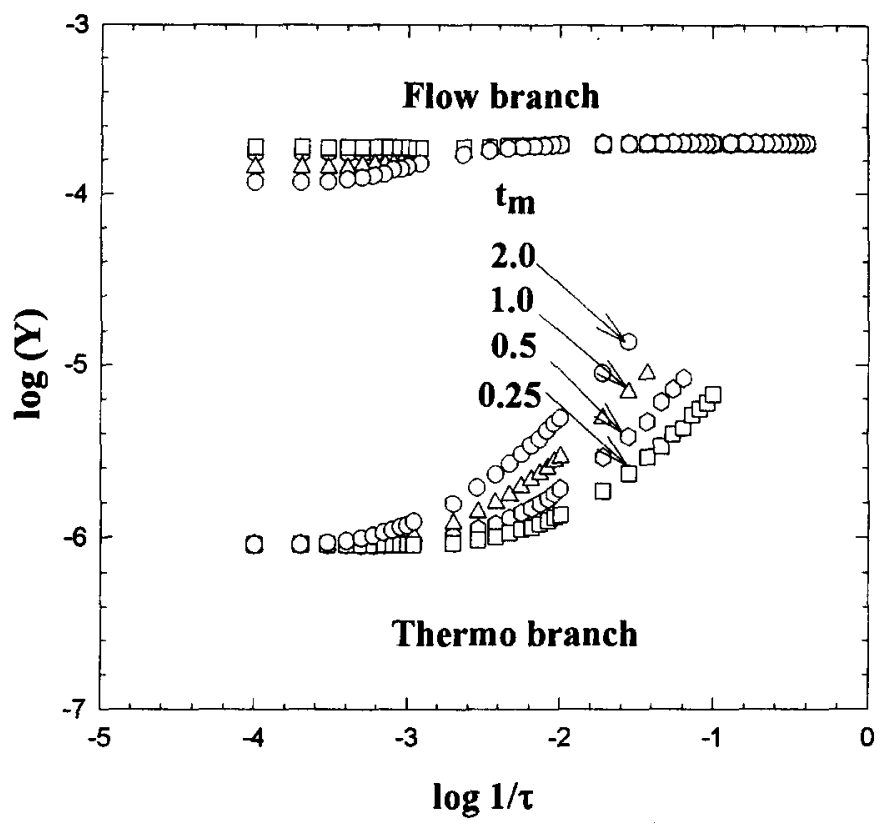

Figure 1. Stability diagram for Kumpinsky-Epstein model.

Effects of mixing time.

demonstrated in Fig. 3. The results with $N=500$ and 1000 are close. The periods would approach asymptotically to a plateau value. This result indicates that convergent results can be obtained if the particle number is larger than approximately 500 .

On the other hand, however, as demonstrated by the dashed curves in Fig. 2, no oscillation patterns will be generated from the USIEM model even when the fluid particle number has increased to beyond 2000 with the same parameter set.

From the above two examples, we can thereby conclude that, when comparing the original SSIEM or USIEM model with the random replacement IEM model proposed here, the present model is equivalent to the SSIEM model when chemical kinetics exhibits multiple steady state characteristics, and is superior to USIEM for simulating the stiff chemical systems.

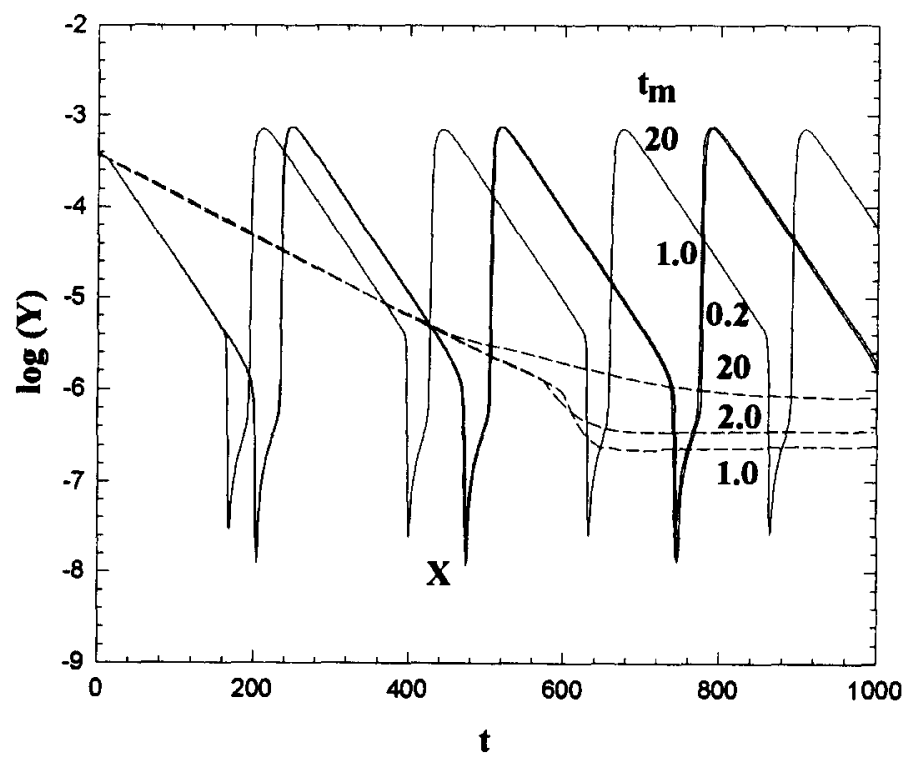

Figure 2. Time evolutions of reactant $Y$ for Oregonator model.

Solid curves: present IEM model; dashed curves: USIEM model. 


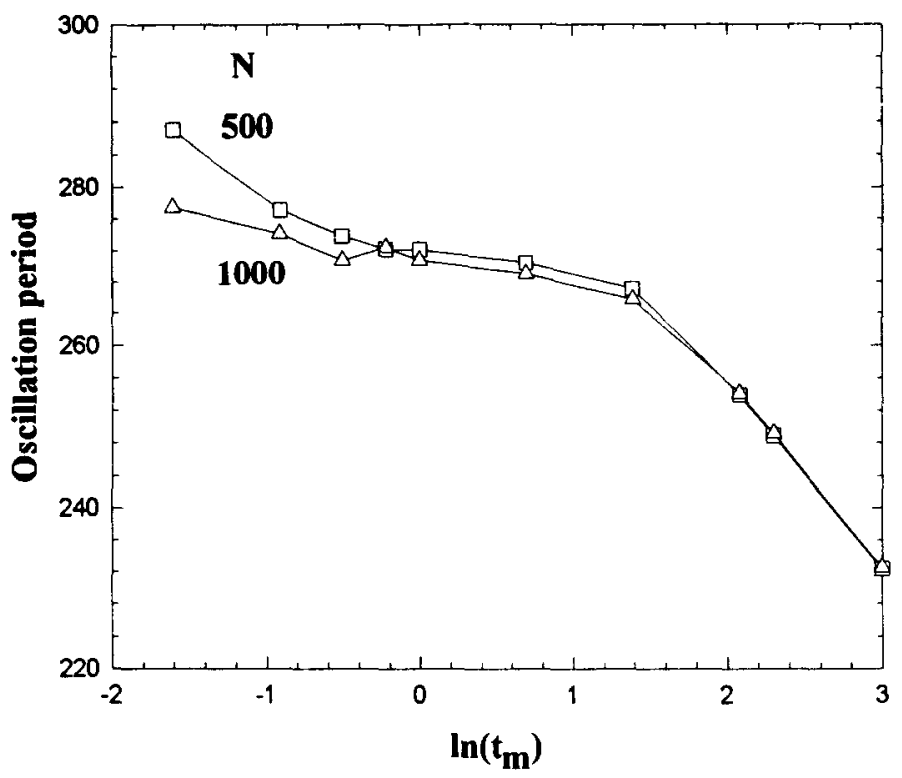

Figure 3. Oscillation periods vs mixing time for Oregonator model.

\section{CONCLUSIONS}

A random replacement IEM model, which considers the mean field concentrations as a direct space average over the existing fluid particles, is proposed as a numerically more favourable IEM scheme. For the steady-state simulations such as the bistable Kumpinsky-Epstein model, the results from the random replacement IEM model agree well with those from the original steady-state IEM model. For chemical systems with a stiff kinetics, nevertheless, the random replacement IEM model is found superior to the unsteady-state IEM model. The oscillation patterns are correctly generated and the oscillation period lengthening phenomena are observed from the present model. On the other hand, the original unsteady-state IEM model fails to generate any oscillation patterns.

ACKNOWLEDGEMENTS - The authors want to thank the financial support from National Science Council, ROC. Part of the numerical works were performed on the IBM SP2 cluster in the National Center for High-Performance Computing, NSC, Taiwan.

\section{NOTATIONS}

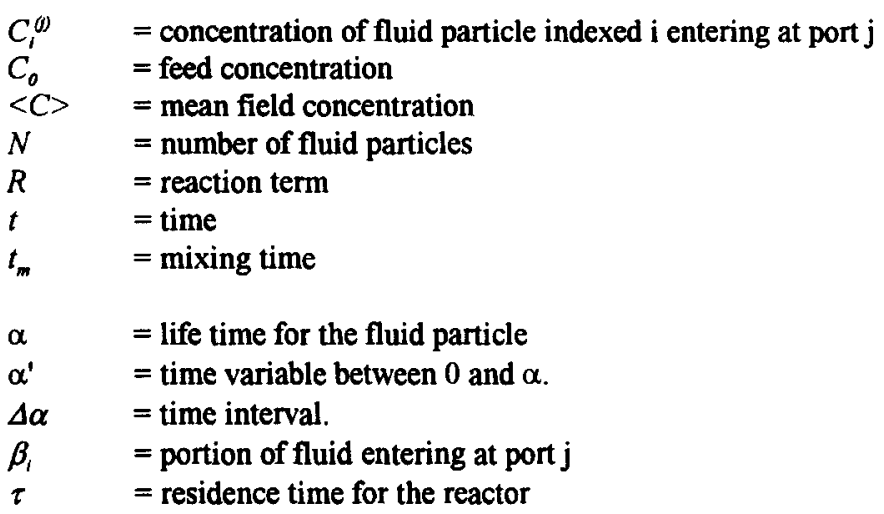

\section{REFERENCES}

Boissonade, J. and De Kepper, P., 1987, A comprehensive interpretation of mixing effects on stationary states and dynamical behavior of the bistable chlorite-iodide reaction in a flow reactor. J. Chem. Phys. 87, 210-218.

Chang, P.C., 1992, Theories for nonlinear chemical dynamic systems. Master thesis, Natl. Taiwan Univ, Taipei, Taiwan.

Dutt, A. K. and Menzinger, M., 1992, Effect of stirring and temperature on the Belousov-Zhabotinskii reaction in a CSTR. J. Phys. Chem. 96, 8447-8449. 
Field, R. J. and Noyes, R. M.,1974, Oscillations in chemical systems. IV. Limit cycle behavior in a model of a real chemical reaction. J. Chem. Phys. 60, 1877-1884.

Fox, R.O. and Villermaux, J., 1990a, Unsteady-state IEM model: numerical simulation and multiple-scale perturbation analysis near perfect-micromixing limit. Chem. Engng. Sci. 45, 373-386

Fox, R.O. and Villermaux, J., 1990b, Micromixing effects in the chlorite-iodide reaction. Chem. Engng. Sci. 45, 2857-2876.

Fox, R.O., Erjaee, G. and Zou, Q., 1994, Bifurcation and stability analysis of micromixing effects in the chlorite-iodide reaction. Chem. Engng. Sci. 49, 3465-3484.

Gyorgyi, L. and Field, R.J., 1992, Simulation of the effect of stirring rate on bistability in the $\mathrm{BrO}_{3}-\mathrm{Ce}$ (III)-Br CSTR reaction. J. Phys. Chem. 96, 1220-1224.

Horsthemke, W. and Hannon, L., 1984, Nonequilibrium chemical instabilities in continuous flow stirred tank reactor: the effects of stirring. J. Chem. Phys. 81, 4363-4368.

Hsu, T. J. and Lee, D. J., 1995, Macromixing effects on the Kumpinsky-Epstein model of a chlorite-iodide reaction in a stirred tank. J. Chem. Phys. 102, 8274-8276.

Hsu, T. J., Mou, C. Y. and Lee, D. J., 1994, Effects of macromixing on the Oregonator model of the Belousov-Zhabotinsky reaction in a stirred tank. Chem. Engng. Sci. 24B, 5291-5305.

Hsu, T. J., Mou, C. Y. and Lee, D. J., 1995, Macromixing effects on the Gray-Scott model in a stirred reactor. Chem. Engng Sci. in press.

Kumpinsky, E. and Epstein, I. R., 1985, A model for stirring effects on transitions in bistable chemical systems. $J$. Chem. Phys. 82, 53-57.

Menzinger, M., Boukalouch, M., De Kepper, P., Boissonade, J., Roux, J. C. and Saadaoni, H., 1986, Dynamic consequences of nonideal mixing in continuously stirred tank reactor studies of chemical instabilities: comparative stirring effects of premixed and nonpremixed feeds on the bistable $\mathrm{ClO}_{2}^{-}+\mathrm{I}^{-}$reaction. $J$. Phys. Chem. 90, 313-315.

Puhl, A. and Nicolis, G., 1986, Micromixing and multiple steady state transition in a CSTR. Chem. Engng. Sci. 41, 3111-3117.

Paul, A. and Nicolis, G., 1987, Normal form analysis of multiple bifurcations in incompletely mixed chemical reaction. J. Chem. Phys. 87, 1070-1078.

Rouff, P., 1993, Excitations induced by fluctuations: an explanation of stirring effects and chaos in closed anaerobic classical Belousov-Zhabontinsky systems. J. Phys. Chem. 97, 6405-6411.

Villermaux, J., 1991, Mixing effects on complex chemical reactions in a stirred reactor. Rev. Chem. Engng., 7, 51-108. 